\title{
Conservative versus surgical management of Pipkin type I fractures associated with posterior dislocation of the hip: a randomised controlled trial
}

\author{
Sujit Kumar Tripathy • Ramesh Kumar Sen • \\ Tarun Goyal
}

Received: 28 July 2011 / Accepted: 22 August 2011 / Published online: 17 September 2011

(C) Springer-Verlag 2011

To the editor,

We read the article by Chen et al. [2] with great interest. In this randomised controlled trial, the authors concluded that closed reduction followed by surgical fragment excision for Pipkin type 1 fractures is better than closed reduction alone. However, we have several concerns regarding the treatment methods and their outcome evaluation in this study.

Before discussing the article by Chen et al., we briefly describe the current strategy for Pipkin fracture-dislocation management focusing mainly on type 1 fracture [1]. The philosophy of Epstein in the management of femoral-head fracture is no longer accepted. Epstein believed that closed reduction was contraindicated for fracture dislocation of the hip when open reduction is planned later. In contrast, current management strategy consists of urgent closed reduction under sedation, preferably within six hours of injury, to minimise chances of avascular necrosis, irrespective of future surgical plans [1, 4]. A proper evaluation of the radiographs before reduction is necessary to exclude hairline or undisplaced femoral-neck fracture. Again, the reduction manoeuvre should be as gentle as possible to avoid iatrogenic complications. All hips after reduction should be assessed with radiographs and computed tomographic (CT) scan (2-mm slice) to assess joint congruency. If the joint is congruent and stable, conservative management with traction should be continued for at least six weeks. Subsequent

S. K. Tripathy $\cdot$ R. K. Sen $\cdot$ T. Goyal

Dept of Orthopaedics,

Postgraduate Institute of Medical Education and Research,

Chandigarh 160012, India

S. K. Tripathy $(\triangle)$

Dept of Orthopaedics, Friarage Hospital,

Northallerton, United Kingdom DL6 1JG

e-mail: sujitortho@yahoo.co.in mobilisation depends on fracture healing on radiographs. Failure to achieve closed reduction, fracture gap $\geq 2 \mathrm{~mm}$ and presence of intra-articular fragments or debris are indications for surgical intervention. Ultimately, the aim of treatment is anatomical reduction of the fracture to restore articular congruency $[1,4]$. If we consider these treatment philosophies, the article in question seems to be lacking in many aspects.

First of all, it is difficult to randomise such studies. In their article, the authors randomly selected patients and assigned them to either the conservative or the surgical group. A patient assigned to the conservative group may need surgical intervention if a congruous joint is not achieved. In such a situation, randomisation will not be possible. The authors were fortunate that they could achieve acceptable closed reduction in all cases in the conservative group.

The authors found no significant difference between groups in relation to age, sex and time between injuries to reduction; even the postoperative protocol was the same in both groups. Thus, no extra benefit in terms of early mobilisation in the postoperative period was gained by the surgical group despite surgical excision of the fragment. We could not understand why surgical patients were immobilised for such a long time (12 weeks) after fragment excision (it is not a fixation). Three-week immobilisation should have been sufficient to allow soft-tissue healing. After a similar time of follow-up, a significantly better outcome was observed in the surgically treated group. In the conservative group, one patient had excellent, three had good, two had fair and two had poor outcomes according to the Thompson and Epstein score and the Merle d'Aubigne and Postel score. In the surgical group, five were excellent, two were good, one was fair and none was graded as poor. The authors noticed avascular necrosis 
(AVN) in two cases (both had poor outcome) and heterotopic ossification (HO) in one case in the conservative group. On the other hand, four cases in the surgical group developed HO. As per the literature, the poor outcome of Pipkin fracture dislocation is largely dependent on complications [3]. We were not able to understand why AVN was attributed to the "conservative management plan". It could, in fact, be because of the initial severity of trauma and hip dislocation, unless a vigorous closed reduction was attempted and had compromised vascularity $[1,3,4]$. It is true that the surgical approach may cause AVN, which may contribute to poor outcome in surgically treated patients [3]. Can the authors confirm that the four cases of $\mathrm{HO}$ in the surgical group were not attributable to surgical intervention? Again, can they confirm the AVN in the conservative group is because of the conservative management plan? If not, then we are certain that the surgical group definitely had some complication because of surgery. On the other hand, perhaps the complications seen in the conservative group were not due to treatment plan but rather to the severity of initial injury. If we exclude the two AVN patients from the conservative group, four (67\%) of the remaining six patients had excellent to good outcome. With these figures, we do not believe that the surgical group would show a significantly better outcome on statistical analysis.

Kloen et al. [4] specifically mentioned that closed anatomical reduction in Pipkin I and II is the best treatment option. If this cannot be obtained (as determined on postreduction CT), osteosynthesis is likely to lead to a better result than is excision. As per the literature, closed reduction leads to excellent/good results for Pipkin I and II fractures in about $75 \%$ of cases, open reduction and internal fixation (ORIF) yields similar results in $64 \%$ of cases and fragment excision gives good/excellent results in only $50 \%$ of cases. Our experience after treating over 100 cases of Pipkin fracture dislocation is not apparently different from that mentioned in the literature. We noted equivalent or even better outcome in conservatively treated patients with Pipkin fracture-dislocation compared to surgical patients (excision or osteosynthesis). Epstein advocated fragment excision, stating that up to one third of the non-weightbearing portion of the femoral head can be excised without compromising the function. However, maintaining joint congruity is a prerequisite for a good outcome, which is reinforced by the more recent literature $[1,3,4]$. We believe that the article by Chen et al. needs greater clarification before their philosophy regarding Pipkin fracture-dislocation management is accepted over the long standing trends of management. Randomisation is not the strength of their article for reasons discussed above. Rather, the small series, with lack of scientific explanation for better outcome in surgically treated patients, is definitely its shortcoming.

The authors discussed Henle's [3] perception in treating such injury and mentioned that closed reduction is very difficult in Pipkin fracture dislocation. Henle could achieve only one proper closed reduction in his series of 12 cases. The remaining cases needed surgical intervention for reduction. Surprisingly, that was not the case in Chen et al.'s article, in which it was reported that they could achieve good reduction in all 16 cases. Biomechanical cadaveric studies indicate that excision of a small part $(<1 / 3)$ of the non-weight-bearing surface of the femoral head (as for Pipkin 1) does not lead to adverse long-term clinical implications. However, these cadaveric studies only consider the force acting on the hip joint but do not consider in vivo surgical morbidity, soft-tissue handling and consequences of the surgical approach. Again, the authors provided no information regarding the size of excised fragments in surgically treated patients.

Considering the above limitations, we do not believe that the concept of the article is clear or would contribute towards a management plan of Pipkin fracture dislocation type I. Further clarification from the authors will be requested before accepting this article as an initiative in a new philosophy of Pipkin fracture-dislocation management.

\section{References}

1. Asghar FA, Karunakar MA (2004) Femoral head fractures: diagnosis, management, and complications. Orthop Clin N Am 35:463-472

2. Chen Z, Bin Lin B, Zhai W, Guo Z, Liang Z, Zheng JP, Lian K, Ding Z (2011) Conservative versus surgical management of Pipkin type I fractures associated with posterior dislocation of the hip: a randomised controlled trial. International Orthopaedics (SICOT) 35:1077-1081

3. Henle P, Kloen P, Siebenrock KA (2007) Femoral head injuries: Which treatment strategy can be recommended? Injury 38:478-488

4. Kloen P, Siebenrock KA, Raaymakers ELFB, Marti RK, Ganz R (2002) Femoral Head Fractures Revisited. Eur J Trauma 28:22133. doi:10.1007/s00068-002-1173-4 